\title{
Glycometabolic Control and Fibrinolysis in Diabetic Patients
}

\begin{tabular}{|l|l|l|}
\hline J.W.J. & & van Wersch $^{\mathrm{a}}$ \\
\hline L.W.J.J.M. & & Westerhuis $^{\mathrm{b}}$ \\
\hline W.J.R.R. & & Venekamp $^{\mathrm{b}}$ \\
\hline
\end{tabular}

${ }^{a}$ De Wever Hospital, Heerlen, ${ }^{b}$ St. Gregorius Hospital, Brunssum, The Netherlands J.W.J. van Wersch, PhD, De Wever Hospital, NL-6401 CX Heerlen (The Netherlands)

\section{Dear Sir,}

We received the comments of Semra et al. on the above article [1]. The commentators argue that the process of fibrinolysis is still much more complicated than being the result of the tissue-type plasminogen activator and plasminogen activator inhibitor (PAI-1) balance, as PAI-1 is also inhibited by protein C. Of course, we agree on this point. But Semra et al. state that consideration of D-dimer levels is not enough for a judgement of the rate of fibrinolysis. We think, however, that it is out of doubt that the $\mathrm{Z}>$-dimer levels are the ultimate signs of previous fibrin formation and fibrin lysis. Thus the measurement of the $\mathrm{Z}>$-dimer levels is in our view the final net result of the process of fibrinolysis. We do not want to oppose the proposal to take the euglobolin lysis time assay, but to our knowledge the D-dimer assay is known to possess a higher reproducibility and a higher sensitivity. So we think that the D-dimer test is the most suitable test for the investigation of the net fibrinolytic effect in a test system.

Regarding age matching of the control group with the patient group, we too are well aware of this fact. The control group used in our study should, however, not be seen as a control group in the strict sense of the word, but rather as a group of subjectively healthy individuals used to calculate the reference range for the different parameters therefrom and to estimate the deviations of the patient group against this reference group. We know that this is a problem, but we do not think that it should have been overcome by multi-variate regression analysis. To compose a correctly designed control group for this complex patient group remains difficult.

With regard to the use of the significant positive correlation between PAI and HbAlc, we wish to point out that we only have stated carefully that the metabolic state may have consequences for the fibrinolysis.

Finally, the recommendation to study the same patients with and without good glycometabolic control seems to us to be impracticable. Such a study design would be outside of the guidelines of the medical ethical committee functioning in our hospital.

Reference 1 van Wersch JWJ, Westerhuis LWJJM, Venekamp WJRR: Glycometabolic control and fibrinolysis in diabetic patients. Haemostasis 1990;20:241-250. 